\title{
Overexpression of CAP1 and its significance in tumor cell proliferation, migration and invasion in glioma
}

\author{
YUE-CHAO FAN $^{1 *}$, CHEN-CHEN CUI $^{1 *}$, YI-SHUO ZHU ${ }^{1,2}$, LEI ZHANG $^{1}$, MENG SHI $^{1}$, \\ JIN-SONG YU ${ }^{1}$, JIN BAI ${ }^{2,3}$ and JUN-NIAN ZHENG ${ }^{2,3}$
}

\author{
${ }^{1}$ Department of Neurosurgery, The Affiliated Hospital of Xuzhou Medical College, Xuzhou, Jiangsu 221002; \\ ${ }^{2}$ Jiangsu Key Laboratory of Biological Cancer Therapy, and ${ }^{3}$ Jiangsu Center for the Collaboration and \\ Innovation of Cancer Biotherapy, Cancer Institute, Xuzhou Medical College, Xuzhou, Jiangsu 221002, P.R. China
}

Received February 24, 2016; Accepted April 19, 2016

DOI: $10.3892 /$ or.2016.4936

\begin{abstract}
Adenylate cyclase-associated protein 1 (CAP1), a protein related to the regulation of actin filaments and the Ras/cAMP pathway, is associated with tumor progression. Nevertheless, the expression level and effects of CAP1 in regards to glioma have not been reported. In the present study, we examined the expression of CAP1 in glioma and tumor adjacent normal brain tissues by tissue microarray and immunohistochemistry. Our results showed that CAP1 was overexpressed in glioma tissues in comparison with that noted in the tumor adjacent normal brain tissues and increased staining of CAP1 was found to be correlated with WHO stage. In addition, we discovered that knockdown of CAP1 by specific RNA interference markedly inhibited cell growth and caused downregulation of the proliferation markers, PCNA and cyclin A. We further demonstrated that knockdown of CAP1 inhibited cell metastatic abilities by downregulating $\mathrm{N}$-cadherin and vimentin and upregulating E-cadherin. These findings revealed that CAP1 expression is markedly increased in human glioma and that downregulation of CAP1 in tumors may serve as a treatment for glioma patients.
\end{abstract}

\section{Introduction}

Glioma is the most common primary intracranial tumor that arises from the neuroectoderm, and thereby is also known as neuroectodermal or neuroepithelial tumor, and accounts for $50 \%$ of primary intracranial tumors $(1,2)$. As the most aggressive type of glioma, glioblastoma multiform (GBM) is a grade IV

Correspondence to: Professor Jun-Nian Zheng or Professor Jin Bai, Jiangsu Key Laboratory of Biological Cancer Therapy, Xuzhou Medical College, 84 West Huai-Hai Road, Xuzhou, Jiangsu 221002, P.R. China

E-mail: jnzheng@xzmc.edu.cn

E-mail: bj@xzmc.edu.cn

*Contributed equally

Key words: CAP1, glioma, proliferation, migration, invasion histological malignancy according to the WHO classification, with a median patient survival period of 12-14 months (3). The poor prognosis of glioblastomas is largely attributed to their rapid growth, high invasive and migratory abilities and high rate of recurrence (4). Therefore, a comprehensive understanding of the molecular mechanisms which expedite glioma tumor proliferation, migration and invasion is urgently needed in order to develop more effective therapies against glioma.

It is known in cell physiology that suppression of tumor cell metastasis includes regulation of cell-cell junctions, cytoskeletal structure and cell morphology. The actin cytoskeleton is pivotal in tumor cell motility and transition, as its remodeling regulates important cellular processes, such as cell adhesion, metastasis and morphological transformation (5-8). Cyclaseassociated protein 1 (CAP1) was initially cloned from budding yeast and is located in the downstream of the ras gene (9). The $\mathrm{N}$-terminal region of CAP binds to Cyrl and is associated with Ras responsiveness in yeast (10-12). The C-terminal region of this protein binds monomeric actin with high affinity, and is necessary for normal cellular morphology (13) Previous research has shown that CAP1 is overexpressed in hepatocellular carcinoma (14), breast (15) and lung cancer (16), and esophageal squamous cell carcinoma (17). Yet, there is sparse research on the influence of CAP1 in gliomas.

In the present study, to evaluate the relationship between the expression of CAP1 and clinicopathological features of glioma, we used a tissue microarray (TMA) of human glioma patient tissues and immunohistochemistry. We discovered that CAP1 expression was obviously upregulated in the glioma tissues when compared with that noted in the tumor adjacent normal brain tissues and this increased expression was correlated with WHO stage. We found that CAP1 was markedly upregulated in glioma tissues when compared to that in normal brain tissues. We then demonstrated that knockdown of CAP1 suppressed glioma cell proliferation, migratory and invasive abilities in glioma cells. We also investigated the molecular mechanisms of CAP1 in glioma cells.

\section{Materials and methods}

Patients and samples. We purchased a glioma TMA kit from Shanxi Alenabio Biotechnology (Xi'an, Shanxi, China) 
(no. GL2083a). Based on WHO criteria, the tumors were divided according to the pathological grade as follows: 134 cases with grades I-II and 58 cases with grades III-IV. Furthermore, it included 8 cases of normal brain tissue and 8 cases of tumor adjacent normal brain tissue. The array dot diameter was $1.0 \mathrm{~mm}$, and each dot represented a tissue spot from one individual specimen that was selected and pathologically confirmed.

Immunohistochemistry of TMA. TMA slices were dewaxed at $62^{\circ} \mathrm{C}$ for $2 \mathrm{~h}$, followed by two 30-min washes with xylene. The sections were blended by 5 -min washes in 100, 95 and $80 \%$ ethanol and sequentially distilled water. Using a microwave the citrate buffer ( $\mathrm{pH} \mathrm{6.0)}$ was heated to $95^{\circ} \mathrm{C}$, and then the sections were placed in the buffer for $15 \mathrm{~min}$. The citrate buffer was cooled to room temperature after the restoration. Phosphate-buffered saline (PBS) was washed out by three 5-min washings, and then hydrogen peroxide was added to the slices for $30 \mathrm{~min}$ in order to block endogenous peroxidase activity. After incubating the sections for 30 min with $10 \%$ goat serum, the sections were incubated with monoclonal rabbit anti-CAP1 antibody (1:500) (Abcam, Cambridge, MA, USA) at $4^{\circ} \mathrm{C}$ overnight. On the following day, the slices were removed at $4^{\circ} \mathrm{C}$ and rewarmed at room temperature for $30 \mathrm{~min}$, and PBS was washed out by three washings for $5 \mathrm{~min}$. Then, the sections were incubated with a biotinylated secondary antibody at room temperature for $30 \mathrm{~min}$, followed by incubation with streptavidin-peroxidase (both from Zhongshan Biotech, Beijing, China) for an additional $30 \mathrm{~min}$. After rinsing with PBS 3 times for $5 \mathrm{~min}$, the sections were stained using DAB (Zhongshan Biotech), rinsed in distilled water and counterstained with hematoxylin. Dehydration was then performed in 80, 95, 95 and $100 \%$ ethanol and distilled water sequentially. Then, the sections were sealed with coverslips. Negative controls were stained with non-immune serum to replace primary antibodies.

Evaluation of immunohistochemical staining. The evaluation of CAP1 staining was blindly and independently examined by the intensity of staining and the proportion of tumor cells showing an unequivocal positive reaction. The CAP1 staining intensity was scored as $0-3$ ( 0 , negative; 1 , weak; 2 , moderate; and 3, strong). The proportion of CAP1-positive stained cells was also scored according to 4 categories: 1 (0-25\%), 2 (26-50\%), $3(51-75 \%)$ and 4 (76-100\%). For statistical analyses, the level of CAP1 staining was evaluated by the immunoreactive score (IRS), which was calculated by multiplying the score of the staining intensity and the proportion of positive cells. According to the IRS, the CAP1 staining pattern was categorized as negative (IRS, 0), weak (IRS, 1-3), moderate (IRS, 4-6) and strong (IRS, 8-12).

Cell culture and transfection. U251 and U87 human glioblastoma cell lines were originally obtained from the Shanghai Institute of Biochemistry and Cell Biology, Chinese Academy of Sciences (Shanghai, China). The cells were cultivated in high glucose Dulbecco's modified Eagle's medium (DMEM) with 10\% fetal bovine serum (FBS) (Invitrogen, Shanghai, China) in a $5 \% \mathrm{CO}_{2}$ humidified atmosphere at $37^{\circ} \mathrm{C}$. When cells grew to $50 \%$ confluency, they were then transfected. Non-specific control siRNA or CAP1 siRNA (both from Qiagen, Mississauga, ON, Canada) were transfected with siLentFect lipid reagent (Bio-Rad, Hercules, CA, USA) according to the manufacturer's instructions. The medium embodying the transfection reagents was replaced after $24 \mathrm{~h}$. Then, the cells were irrigated twice with PBS and maintained in fresh medium. Forty-eight hours after transfection, the cells were lysed for western blot assay and were used for CCK-8 cell proliferation, cell migration and Matrigel invasion assays.

Western blot analysis. Treated and untreated cells were lysed in radioimmunoprecipitation assay buffer with a freshly added protease inhibitor cocktail (Roche Applied Science, Indianapolis, IN, USA). The protein density was determined using the bicinchoninic acid (BCA) assay (Pierce, Rockford, IL, USA). All protein samples were formulated and denatured. Protein was separated by SDS-PAGE on $8-12 \%$ gradient polyacrylamide gel and transferred onto polyvinylidene difluoride membranes (Millipore, Billerica, MA, USA). The membranes were blocked in 5\% non-fat milk for $3 \mathrm{~h}$ at room temperature, and then incubated with the primary antibody overnight at $4^{\circ} \mathrm{C}$. The following antibodies were applied: rabbit anti-CAP1 (Abcam), rabbit anti-N-cadherin, rabbit anti-vimentin, rabbit anti-E-cadherin (all from Cell Signaling Technology, Beverly, MA, USA), rabbit anti-PCNA and rabbit anti-cyclin A (Santa Cruz Biotechnology, Inc., Santa Cruz, CA, USA) and rabbit anti- $\beta$-actin (Cell Signaling Technology). The membranes were then incubated with peroxidase-conjugated Affinipure goat anti-rabbit IgG secondary antibody (Zhongshan Biotech, Beijing, China) for $2 \mathrm{~h}$ at room temperature. Detection was performed using an enhanced chemiluminescence method (Pierce).

Cell growth assay. Cell growth was analyzed using the Cell Counting Kit-8 (CCK-8; Beyotime, Nantong, China). U87 and $\mathrm{U} 251$ cells were cultured at a density of $5 \times 10^{3}$ cells/well and suspended in $100 \mu \mathrm{l}$ high glucose DMEM containing $10 \%$ FBS in 96-well plates and incubated for 1, 2, 3 and 4 days, respectively. Then, $10 \mu \mathrm{l}$ CCK- 8 solution was added to each well and incubated at $37^{\circ} \mathrm{C}$ for $1 \mathrm{~h}$. The optical density was measured at $450 \mathrm{~nm}$ on an ELX-800 spectrometer reader (Bio-Tek Instruments, Winooski, VT, USA).

Wound-healing assay. Cells were seeded into 6-well plates at a density of $5 \times 10^{5}$ cells/well in culture medium and transfected with siRNA. Twenty-four hours after transfection, the cells were scratched on the monolayer with a $10-\mu 1$ pipette tip and washed twice with PBS. Then, the cells were maintained in high glucose DMEM for an additional $24 \mathrm{~h}$. Images were captured using an inverted Leica phase-contrast microscope (Leica DFC300 FX) at 0 and $48 \mathrm{~h}$ time points. The percentage of wound healing of the cells was determined by the ratio of the healing width at each time point to the wound width at $0 \mathrm{~h}$.

Migration assay. Cell migration was determined using a modified two-chamber plate with pore size of $8-\mu \mathrm{m}$. For the cell migration assay, $1 \times 10^{5} \mathrm{U} 251$ and U87 cells were seeded in serum-free medium in the upper chamber. To stimulate migration, $10 \%$ of serum containing culture medium was added 


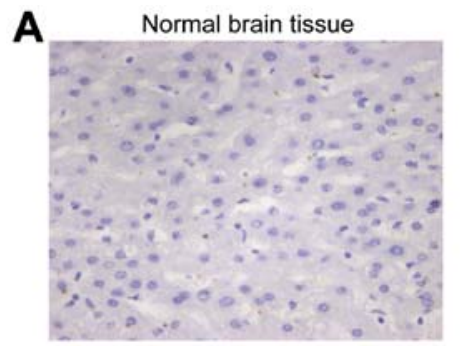

B Tumor adjacent normal brain tissue
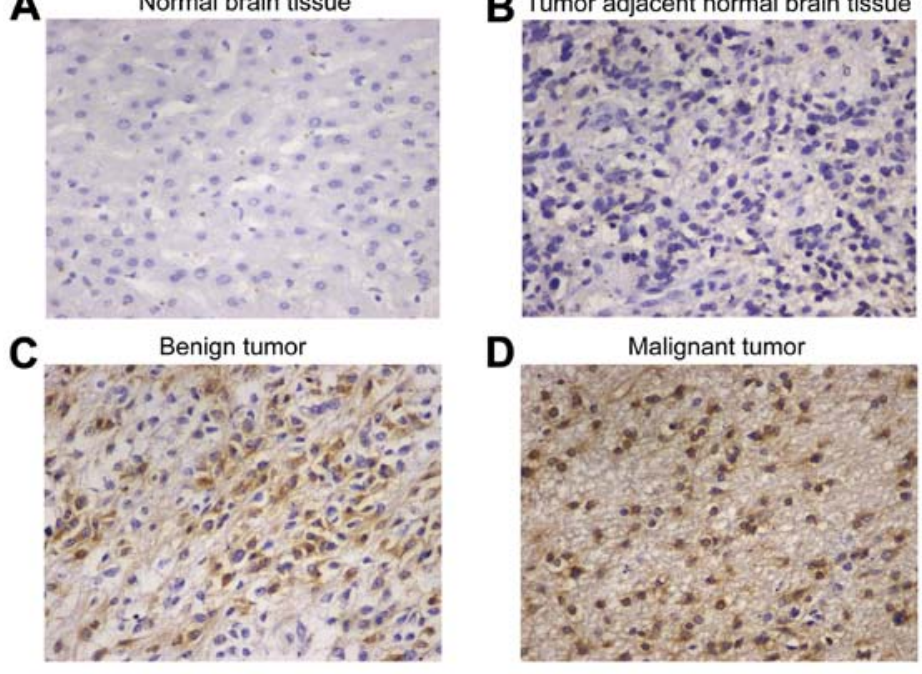

$\mathbf{E}$
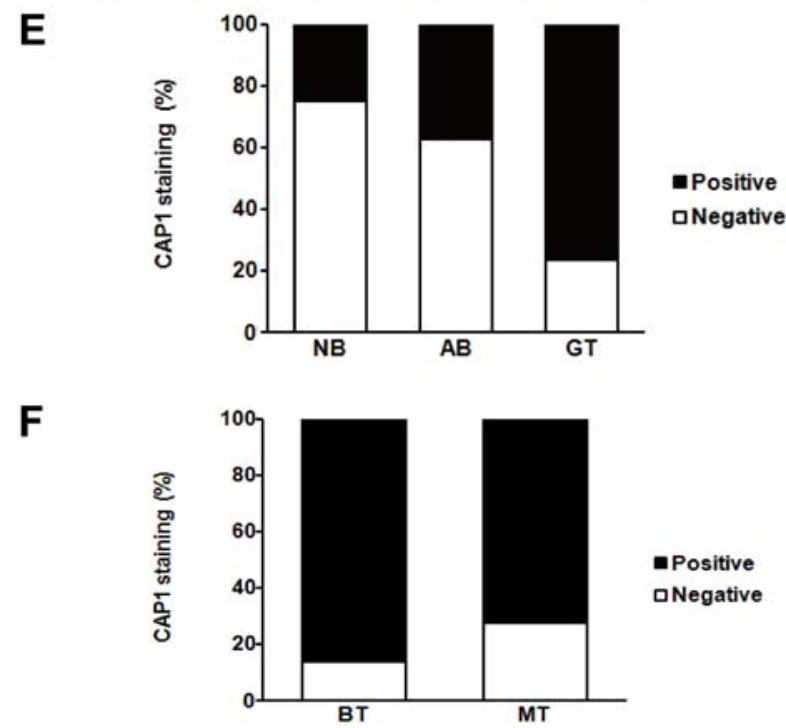

Figure 1. Representative images showing CAP1 immunohistochemical staining. (A) Negative CAP1 staining in normal brain tissue (NB). (B) Negative CAP1 staining in tumor adjacent normal brain tissue (AB). (C) Positive CAP1 staining in benign tumor (BT). (D) Positive CAP1 staining in malignant tumor (MT). (E) A significant difference in CAP1 staining was observed between normal brain tissue and glioma tissue (GT) (P<0.05; $\chi^{2}$ test) and between tumor adjacent normal brain tissue and glioma tumor $\left(\mathrm{P}<0.05 ; \chi^{2}\right.$ test). (F) CAP1 staining was markedly increased in malignant tumor compared with benign tumor $(\mathrm{P}<0.05)$. All experiments were carried out in triplicate. Data are shown as mean \pm SE; (A-D) Original magnification, $x 40$.

to the bottom wells. After the cell was incubated for $24 \mathrm{~h}$ at $37^{\circ} \mathrm{C}$, cells on the top surface of the insert were carefully removed with a cotton swab and the cells that had traversed the membrane were fixed in methanol, stained with crystal violet to visualize nuclei and the number of migrating cells was counted under a magnification of x200 in 5 fields (up, down, median, left and right), and the means for each chamber were determined.

Invasion assay. The invasion assay was performed using a modified two-chamber plate with a pore size of $8-\mu \mathrm{m}$. The Transwell filter inserts were coated with Matrigel, and $1 \times 10^{5} \mathrm{U} 251$ and U87 cells were seeded in serum-free medium in the upper chamber. To stimulate invasion, $10 \%$ of serum containing culture medium was added to the bottom wells. After $24 \mathrm{~h}$ of incubation at $37^{\circ} \mathrm{C}$, the cells in the upper chamber were carefully removed with a cotton swab and the cells that had traversed the membrane were fixed in methanol, stained with Giemsa and counted. For counting, the cells in 5 fields (up, down, median, left and right; magnification, x200) per filter were counted under a microscope.

Statistical analysis. Statistical analysis was carried out using SPSS 19.0 software (SPSS, Inc., Chicago, IL, USA). Values are expressed as the means \pm SD. The relationship between CAP1 staining and the clinicopathological parameters of the glioma patients, such as age, gender, WHO grade and histological type was assessed by $\chi^{2}$ test. For CCK- 8 cell growth assays, the Student's t-test was used. For migration and invasion assays, the results are expressed as the means $\pm \mathrm{SD}$. Each experiment was performed at least 3 times. Differences were considered significant at a P-value $<0.05$.

\section{Results}

CAP1 expression is upregulated in glioma tissues. To investigate whether differential expression of CAP1 exists in glioma tissues, we applied a TMA to detect the CAP1 
Table I. CAP1 staining and clinicopathological characteristics of the 192 glioma patients.

\begin{tabular}{lrrrr}
\hline & \multicolumn{2}{c}{ CAP1 staining } & & \\
\cline { 2 - 3 } & $\begin{array}{c}\text { Negative } \\
\text { No. } \%)\end{array}$ & $\begin{array}{r}\text { Positive } \\
\text { No. }(\%)\end{array}$ & Total & P-value \\
\hline Age (years) & & & & \\
$<46$ & $22(24.7)$ & $67(75.3)$ & 89 & 0.320 \\
$\geq 46$ & $23(22.3)$ & $80(77.7)$ & 103 & \\
Gender & & & & \\
Male & $27(23.1)$ & $90(76.9)$ & 117 & 0.883 \\
Female & $18(24.0)$ & $57(76.0)$ & 75 & \\
WHO grade & & & & \\
Benign (I-II) & $37(27.6)$ & $97(72.4)$ & 134 & 0.038 \\
Malignant (III-IV) & $8(13.8)$ & $50(86.2)$ & 58 & \\
Histological type & & & & \\
Astrocytoma & $32(24.2)$ & $100(75.8)$ & 132 & 0.951 \\
Glioblastoma & $6(18.2)$ & $27(71.8)$ & 33 & \\
Oligoastrocytoma & $2(28.6)$ & $5(71.4)$ & 7 & \\
Ependymoma & $3(27.3)$ & $8(72.7)$ & 11 & \\
Oligodendroglioma & $2(22.2)$ & $7(77.8)$ & 9 & \\
\hline
\end{tabular}

${ }^{a} \chi^{2}$ test. CAP1, cyclase-associated protein 1 .

expression in normal brain and tumor adjacent normal brain tissues, benign tumors (grade I and II) and malignant tumors (grade III and IV). We found that CAP1 was mainly localized in the cytoplasm, yet it was occasionally observed in the nucleus. Representative images are presented in Fig. 1A-D. CAP1positive staining was detected in 2 of $8(25.00 \%)$ normal brain tissues, 3 of $8(37.50 \%)$ tumor adjacent normal brain tissues and 147 of $192(76.56 \%$ ) glioma tissues (Fig. 1E). A significant difference in CAP1 staining was noted between the normal brain and glioma tissues $\left(\mathrm{P}<0.05 ; \chi^{2}\right.$ test) and between tumor adjacent normal brain and glioma tissues $\left(\mathrm{P}<0.05 ; \chi^{2}\right.$ test).

Correlation of CAP1 expression with clinicopathological parameters. Investigation of the relationship between CAP1 expression and clinicopathological parameters of the glioma cases are summarized in Table I. WHO grade and histological type are known as vital prognostic markers for glioma patients. Samples with IRS 0-3 and IRS 4-12 were classified as having low and high expression of CAP1. We discovered CAP1positive staining in 97 of $134(72.39 \%)$ benign tumor tissues and in 50 of $58(86.21 \%)$ malignant tumor tissues. Moreover CAP1 staining was significantly increased in malignant (grade III and IV) compared with benign tumors (grade I and II) $\left(\mathrm{P}<0.05 ; \chi^{2}\right.$ test; Fig. 1F). However, no obvious correlations were noted between CAP1 expression and other clinicopathological parameters, including patient age $(\mathrm{P}=0.683)$, gender $(\mathrm{P}=0.562)$ and histological type $(\mathrm{P}=0.802)$ (Table $\mathrm{I})$.

Knockdown of CAP1 inhibits glioma cell proliferation in vitro. Owing to the fact that CAP1 expression is signifi-
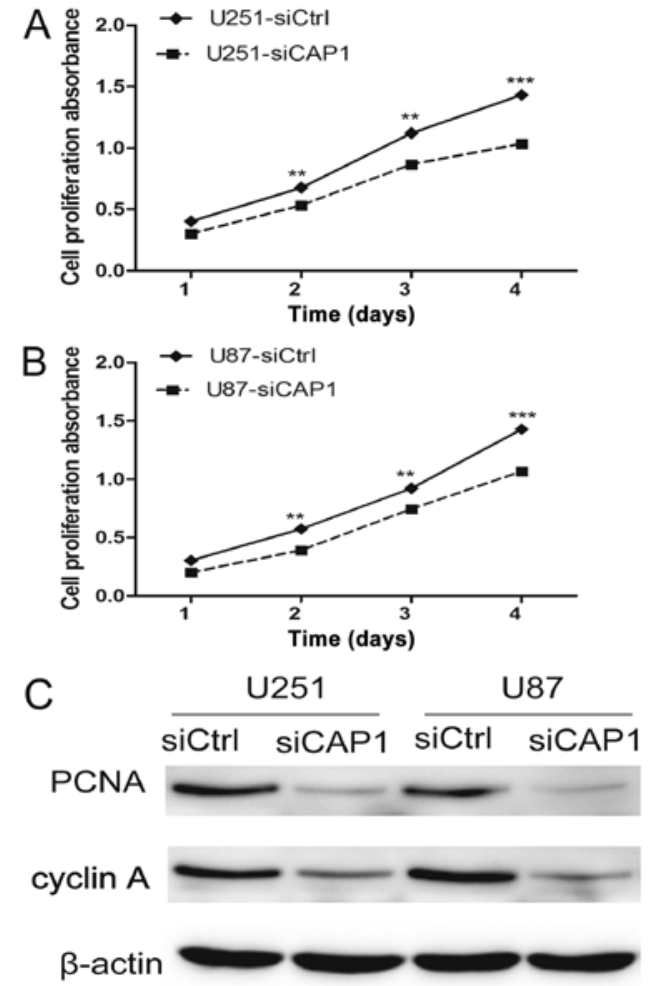

Figure 2. Knockdown of CAP1 inhibits glioma cell proliferation. (A and B) CCK-8 cell proliferation assay was performed after CAP1 knockdown in U251 and U87 cells. (C) Western blot analysis of the relative protein levels of PCNA and cyclin A in CAP1-knockdown and control U251 and U87 cells. All experiments were carried out in triplicate. Data are shown as mean $\pm \mathrm{SE} ;{ }^{* *} \mathrm{P}<0.01,{ }^{* * *} \mathrm{P}<0.001$.

cantly increased in glioma tissues compared with tumor adjacent normal brain tissues, CAP1 may play vital roles in tumor progression. U251 and U87 cells were transfected with siRNA targeting CAP1 to knock down CAP1 expression. The results of the CCK-8 cell proliferation assays revealed decreased growth rates in the U251 and U87 cells depleted of CAP1 (Fig. 2A and B). To determine whether the decreased proliferative ability after CAP1 knockdown was due to alterations in the proliferation markers, PCNA and cyclin A, we performed western blot analysis. Western blot results showed downregulated levels of PCNA and cyclin A in the glioma cells with silenced CAP1 (Fig. 2C).

Knockdown of CAPl inhibits glioma cell migration in vitro. Since actin organization plays a significant role in cell motility and migration, we speculated that CAP1 may exert a crucial function in the regulation of cell motility. To examine this hypothesis, we knocked down the expression of CAP1 in U251 and U87 cell lines and analyzed the role of CAP1 on cell migration by wound healing and Transwell assays. We observed that wound recovery was significantly reduced by 26.58 and $38.75 \%$ after CAP1 knockdown (Fig. 3A and B), and the percentage of cells that transversed through the micropore membrane in the Transwell assay was significantly decreased by 44.44 and $36.21 \%$ (Fig. 3C and D) in the U251 and U87 cells lines as compared with the non-transfected cells. In summary, these results indicated that interference of CAP1 had an evident inhibitory effect on glioma cell motility. 
A

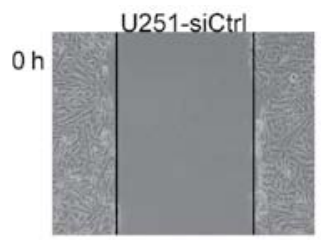

$24 \mathrm{~h}$
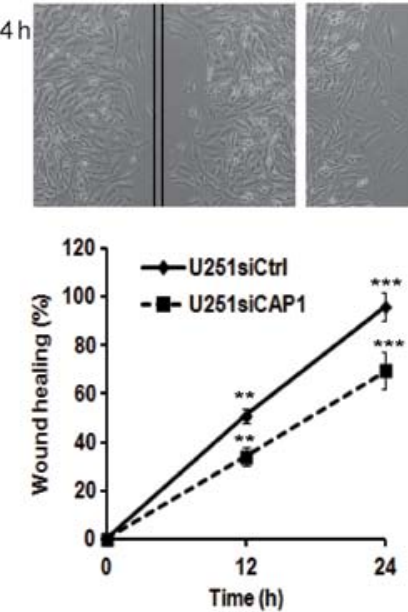

C
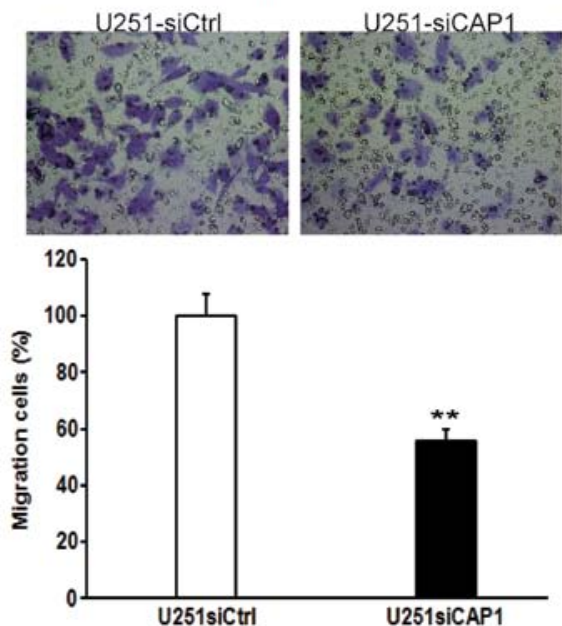
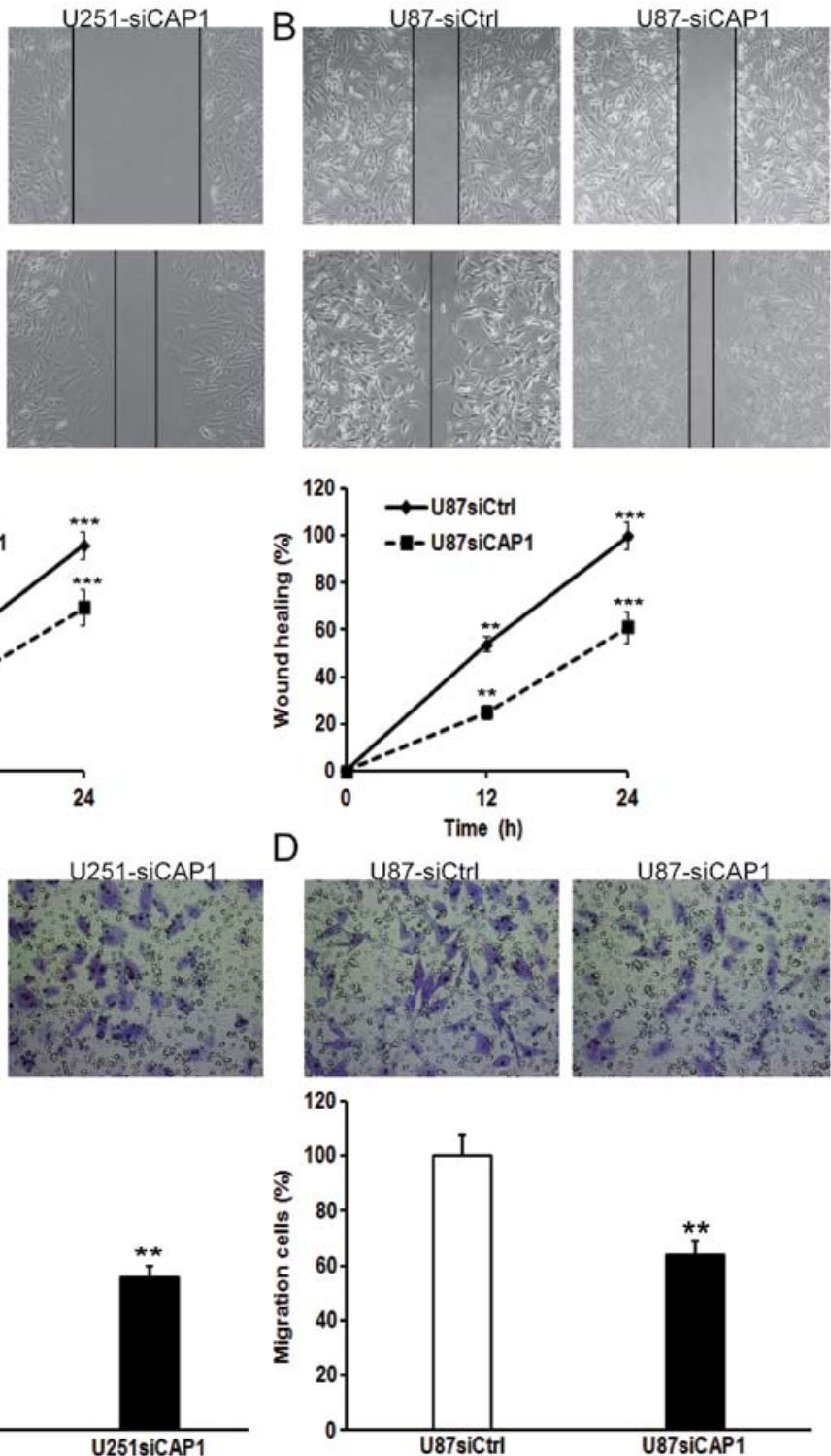

Figure 3. Knockdown of CAP1 inhibits glioma cell motility. (A and B) Wound-healing assay was performed after CAP1 knockdown in U251 and U87 cells. There was significant delay in wound closure after CAP1 knockdown compared with the control cells. (C and D) Cell migration assay was performed after CAP1 knockdown in U251 and U87 cells. U251-siCAP1 cells and U87-siCAP1 cells exhibited decreased ability to migrate through the Boyden chamber when compared to the control cells. All experiments were carried out in triplicate. Data are shown as mean $\pm \mathrm{SE} ;{ }^{* *} \mathrm{P}<0.01,{ }^{* * * *} \mathrm{P}<0.001$.

Knockdown of CAPl inhibits glioma cell invasion in vitro. For the cell invasion assay, downregulation of CAP1 inhibited the cell invasive ability of the U251 and U87 cells by 31.43 and $70.00 \%$, respectively (Fig. 4A and B). To investigate the mechanisms of the regulation of metastasis by CAP1, we utilized western blotting to detect alterations in the protein levels. A decrease in CAP1 resulted in the upregulation of epithelial marker E-cadherin and downregulation of $\mathrm{N}$-cadherin and vimentin (Fig. 4C). Our results indicated that CAP1 expression may expedite migration and invasion by increasing the expression of $\mathrm{N}$-cadherin and vimentin and downregulation of E-cadherin.

\section{Discussion}

Cyclase-associated protein 1 (CAP1) was initially cloned by budding yeast and is located in the downstream of the ras gene (9). CAP consists of 4 domains which are involved in actin binding, adenylyl cyclase association in yeast, SH3 binding and oligomerization, respectively (18). As a monomeric actin binding protein, CAP takes part in cell polarization, the allocation of actin filaments and mRNA in Dictyostelium (19). The expression of CAP has a relationship with an anomalous large cell size, random budding pattern and an anomalous actin allocation in yeast $(10,20)$. The human homologue of CAP1 was initially detected in the early 1990's (21). Both mammal and yeast CAPs interplay with actin (13) and play a part in actin turnover (22). Given the vital role of actin filament restructuring in cell metastasis and the regulatory role of CAP1 in actin filament reorganization $(18,23)$, we hypothesized that CAP1 may function in tumor metastasis. In addition, the functions of CAPs in mammals and other vertebrates are not well known. Although a previous study demonstrated that CAP1 is overexpressed in pancreatic cancers and revealed an 

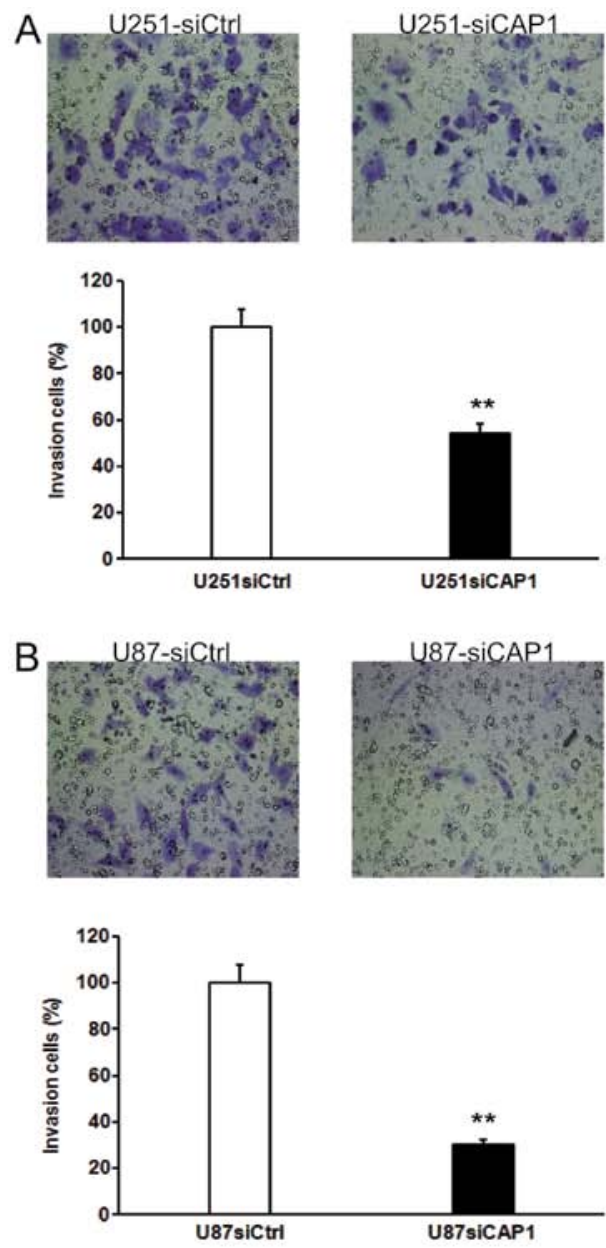

C

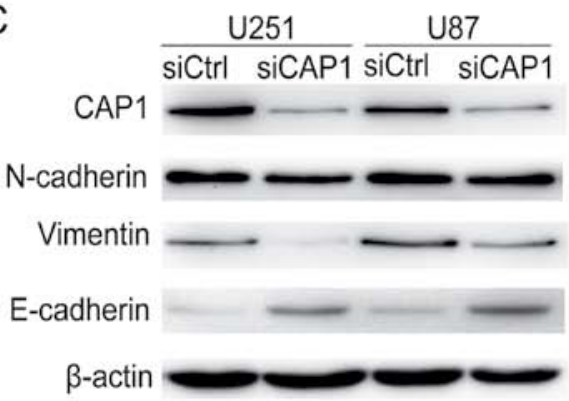

Figure 4. Knockdown of CAP1 inhibits the cell invasion of glioma cells. (A and B) Matrigel cell invasion assay was performed after the knockdown of CAP1 in U251 and U87 cells. (C) Western blot analysis of the relative protein levels of $\mathrm{N}$-cadherin, vimentin and E-cadherin following downregulation of CAP1 and in the control group of U251 and U87 cells. All experiments were carried out in triplicate. Data are shown as mean $\pm \mathrm{SE} ;{ }^{* *} \mathrm{P}<0.01$.

involvement of CAP1 in the aggressive behavior of pancreatic cancer cells (24), the role and molecular mechanism of CAP1 in glioma remain virtually unknown. In the present study, our data demonstrated that CAP1 was obviously increased in glioma tissues compared to tumor adjacent normal brain tissues by immunohistochemical (Fig. 1A-D). Furthermore, CAP1 expression was upregulated in malignant (grade III and IV) compared with benign tumor tissues (grade I and II) (Fig. 1F). This demonstrated that CAP1 may play a vital role in glioma development and progression.

Cyclin A, a major member of the cyclin family, is a component of the machinery required for progression through
$\mathrm{S}$ phase $(25,26)$. Fundamental expression of cyclin A has a relationship with tumorigenesis in diverse studies $(27,28)$. Our results identified a significant decrease in cyclin A expression after silencing of CAP1. However, we found an obvious decrease in the expression of proliferating cell nuclear antigen (PCNA) after silencing of CAP1, an associated protein of DNA polymerase $\delta$. Cyclin A and PCNA were simultaneously downregulated after silencing of CAP1 in the glioma cells. While speculative, these results warrant further study.

Epithelial to mesenchymal transition (EMT) is a step that is conducive to an aggressive cell phenotype which is accompanied by the loss of intercellular adhesion in tumors, leading to the formation of migratory mesenchymal cells with invasive characteristics $(29,30)$. Therefore, EMT is crucial in tumor progression and metastasis (31). In epithelial cells, adhesion molecule, E-cadherin, regulates the interactions between cellcell and tissue framework of organization. The fundamental mechanism involved in the process of EMT is upregulation of the mesenchymal marker vimentin and downregulation of the epithelial marker E-cadherin (32). In the present study, we discovered that downregulation of CAP1 in glioma cells inhibited cell metastatic abilities, which were caused by the downregulation of $\mathrm{N}$-cadherin and vimentin and the upregulation of E-cadherin. However, a more detailed molecular mechanism of how CAP1 regulates the progression of glioma requires further investigation.

In summary, our results demonstrated that CAP1 plays a vital role in human glioma pathogenesis. Overexpression of CAP1 influenced tumor progression by enhancing cell proliferation, migration and invasion. Our results imply that CAP1 may be an important marker and a therapeutic target for gliomas. Thus, the present study may provide a valuable therapeutic strategy to help control the progression of gliomas.

\section{References}

1. Hamza MA and Gilbert M: Targeted therapy in gliomas. Curr Oncol Rep 16: 379, 2014.

2. Kim YZ: Altered histone modifications in gliomas. Brain Tumor Res Treat 2: 7-21, 2014.

3. Wen PY and Kesari S: Malignant gliomas in adults. N Engl J Med 359: 492-507, 2008.

4. Giese A, Bjerkvig R, Berens ME and Westphal M: Cost of migration: Invasion of malignant gliomas and implications for treatment. J Clin Oncol 21: 1624-1636, 2003.

5. Chang E, Heo KS, Woo CH, Lee H, Le NT, Thomas TN, Fujiwara K and Abe J: MK2 SUMOylation regulates actin filament remodeling and subsequent migration in endothelial cells by inhibiting MK2 kinase and HSP27 phosphorylation. Blood 117: 2527-2537, 2011.

6. Kirfel G, Rigort A, Borm B and Herzog V: Cell migration: Mechanisms of rear detachment and the formation of migration tracks. Eur J Cell Biol 83: 717-724, 2004.

7. Hall A: The cytoskeleton and cancer. Cancer Metastasis Rev 28: 5-14, 2009.

8. Fedor-Chaiken M, Deschenes RJ and Broach JR: SRV2, a gene required for $R A S$ activation of adenylate cyclase in yeast. Cell 61: 329-340, 1990.

9. Field J, Vojtek A, Ballester R, Bolger G, Colicelli J, Ferguson K, Gerst J, Kataoka T, Michaeli T, Powers S, et al: Cloning and characterization of $C A P$, the $\mathrm{S}$. cerevisiae gene encoding the $70 \mathrm{kd}$ adenylyl cyclase-associated protein. Cell 61: 319-327, 1990.

10. Gerst JE, Ferguson K, Vojtek A, Wigler M and Field J: CAP is a bifunctional component of the Saccharomyces cerevisiae adenylyl cyclase complex. Mol Cell Biol 11: 1248-1257, 1991.

11. Mintzer KA and Field J: Interactions between adenylyl cyclase, CAP and RAS from Saccharomyces cerevisiae. Cell Signal 6: 681-694, 1994. 
12. Nishida Y, Shima F, Sen H, Tanaka Y, Yanagihara C, YamawakiKataoka Y, Kariya K and Kataoka T: Coiled-coil interaction of N-terminal 36 residues of cyclase-associated protein with adenylyl cyclase is sufficient for its function in Saccharomyces cerevisiae ras pathway. J Biol Chem 273: 28019-28024, 1998.

13. Freeman NL, Chen Z, Horenstein J, Weber A and Field J: An actin monomer binding activity localizes to the carboxyl-terminal half of the Saccharomyces cerevisiae cyclase-associated protein. J Biol Chem 270: 5680-5685, 1995.

14. Liu Y, Cui X, Hu B, Lu C, Huang X, Cai J, He S, Lv L, Cong X, Liu G, et al: Upregulated expression of CAP1 is associated with tumor migration and metastasis in hepatocellular carcinoma. Pathol Res Pract 210: 169-175, 2014.

15. Yu XF, Ni QC, Chen JP, Xu JF, Jiang Y, Yang SY, Ma J, Gu XL, Wang $\mathrm{H}$ and Wang YY: Knocking down the expression of adenylate cyclase-associated protein 1 inhibits the proliferation and migration of breast cancer cells. Exp Mol Pathol 96: 188-194, 2014.-

16. Tan M, Song X, Zhang G, Peng A, Li X, Li M, Liu Y and Wang C: Overexpression of adenylate cyclase-associated protein 1 is associated with metastasis of lung cancer. Oncol Rep 30: 1639-1644, 2013.

17. Li M, Yang X, Shi H, Ren H, Chen X, Zhang S, Zhu J and Zhang J: Downregulated expression of the cyclase-associated protein 1 (CAP1) reduces migration in esophageal squamous cell carcinoma. Jpn J Clin Oncol 43: 856-864, 2013.

18. Hubberstey AV and Mottillo EP: Cyclase-associated proteins: CAPacity for linking signal transduction and actin polymerization. FASEB J 16: 487-499, 2002.

19. Noegel AA, Blau-Wasser R, Sultana H, Müller R, Israel L, Schleicher M, Patel H and Weijer CJ: The cyclase-associated protein CAP as regulator of cell polarity and cAMP signaling in Dictyostelium. Mol Biol Cell 15: 934-945, 2004.

20. Vojtek A, Haarer B, Field J, Gerst J, Pollard TD, Brown S and Wigler M: Evidence for a functional link between profilin and CAP in the yeast S. cerevisiae. Cell 66: 497-505, 1991.

21. Matviw H, Yu G and Young D: Identification of a human cDNA encoding a protein that is structurally and functionally related to the yeast adenylyl cyclase-associated CAP proteins. Mol Cell Biol 12: 5033-5040, 1992.
22. Moriyama $\mathrm{K}$ and Yahara I: Human CAP1 is a key factor in the recycling of cofilin and actin for rapid actin turnover. J Cell Sci 115: 1591-1601, 2002.

23. Loisel TP, Boujemaa R, Pantaloni D and Carlier MF: Reconstitution of actin-based motility of Listeria and Shigella using pure proteins. Nature 401: 613-616, 1999.

24. Yamazaki K, Takamura M, Masugi Y, Mori T, Du W, Hibi T, Hiraoka N, Ohta T, Ohki M, Hirohashi S, et al: Adenylate cyclase-associated protein 1 overexpressed in pancreatic cancers is involved in cancer cell motility. Lab Invest 89: 425-432, 2009.

25. Krek W, Ewen ME, Shirodkar S, Arany Z, Kaelin WG Jr and Livingston DM: Negative regulation of the growth-promoting transcription factor E2F-1 by a stably bound cyclin A-dependent protein kinase. Cell 78: 161-172, 1994

26. Lees E, Faha B, Dulic V, Reed SI and Harlow E: Cyclin E/cdk2 and cyclin A/cdk2 kinases associate with p107 and E2F in a temporally distinct manner. Genes Dev 6: 1874-1885, 1992.

27. Wang J, Chenivesse X, Henglein B and Bréchot C: Hepatitis B virus integration in a cyclin $\mathrm{A}$ gene in a hepatocellular carcinoma. Nature 343: 555-557, 1990.

28. Barlat I, Fesquet D, Bréchot C, Henglein B, Dupuy d'Angeac A, Vié $A$ and Blanchard JM: Loss of the $\mathrm{G}_{1}-\mathrm{S}$ control of cyclin A expression during tumoral progression of Chinese hamster lung fibroblasts. Cell Growth Differ 4: 105-113, 1993.

29. Singh A and Settleman J: EMT, cancer stem cells and drug resistance: An emerging axis of evil in the war on cancer. Oncogene 29: 4741-4751, 2010.

30. Thiery JP: Epithelial-mesenchymal transitions in development and pathologies. Curr Opin Cell Biol 15: 740-746, 2003.

31. Song Y, Washington MK and Crawford HC: Loss of FOXA1/2 is essential for the epithelial-to-mesenchymal transition in pancreatic cancer. Cancer Res 70: 2115-2125, 2010.

32. Huber MA, Kraut $\mathrm{N}$ and Beug $\mathrm{H}$ : Molecular requirements for epithelial-mesenchymal transition during tumor progression. Curr Opin Cell Biol 17: 548-558, 2005. 